\title{
Eye-hand coordination during manual object transport with the affected and less affected hand in adolescents with hemiparetic cerebral palsy
}

\author{
Julius Verrel · Harold Bekkering • Bert Steenbergen
}

Received: 11 June 2007 / Accepted: 14 January 2008 / Published online: 30 January 2008

(C) The Author(s) 2008

\begin{abstract}
In the present study we investigated eye-hand coordination in adolescents with hemiparetic cerebral palsy (CP) and neurologically healthy controls. Using an object prehension and transport task, we addressed two hypotheses, motivated by the question whether early brain damage and the ensuing limitations of motor activity lead to general and/or effector-specific effects in visuomotor control of manual actions. We hypothesized that individuals with hemiparetic CP would more closely visually monitor actions with their affected hand, compared to both their less affected hand and to control participants without a sensorimotor impairment. A second, more speculative hypothesis was that, in relation to previously established deficits in prospective action control in individuals with hemiparetic $\mathrm{CP}$, gaze patterns might be less anticipatory in general, also during actions performed with the less affected hand. Analysis of the gaze and hand movement data revealed the increased visual monitoring of participants with $\mathrm{CP}$ when using their affected hand at the beginning as well as during object transport. In contrast, no general deficit in anticipatory gaze control in the participants with hemiparetic CP could be observed. Collectively, these findings are the first to directly show that individuals with hemiparetic CP adapt eye-hand coordination to the specific constraints of the moving limb, presumably to compensate for sensorimotor deficits.
\end{abstract}

Keywords Congenital brain damage .

Sensorimotor impairment · Visuomotor control ·

Prehension · Adaptation · Affected side

J. Verrel $\cdot$ H. Bekkering $\cdot$ B. Steenbergen $(\bowtie)$

Nijmegen Institute for Cognition and Information,

Radboud University Nijmegen, P.O. Box 9104,

6500 HE Nijmegen, The Netherlands

e-mail: steenbergen@ nici.ru.nl

\section{Introduction}

Cerebral palsy (CP) is an umbrella-term describing a group of disorders of movement and posture leading to activity limitations, and that are caused by damage to the fetal or infant brain (Bax et al. 2005). In this study, we focused on the most common subtype of cerebral palsy, spastic hemiparesis, which typically occurs after unilateral lesions to the cerebral cortex or corticospinal pathways (Kwong et al. 2004). This condition is characterized by impaired control of muscle tone and spasticity in the upper and lower limbs of the contralesional, "affected", side of the body (Albright 1996), generally progressing from proximal to distal (Freund 1987; Steenbergen et al. 2000b), and often accompanied by sensory deficits of proprioception and tactile perception (Cooper et al. 1995). These symptoms induce limitations on manual actions performed with the affected hand $(\mathrm{AH})$, in particular related to fine motor skills such as fingertip force control (Eliasson et al. 1991). These limitations are further amplified by more central deficits in the integration of different modalities, such as matching proprioceptive and visual spatial information (Wann 1991), and integrating sensory information with motor output (Gordon et al. 2006). While the less affected hand (LAH) is usually spared from severe sensorimotor impairments (but see, Steenbergen and Meulenbroek 2006 for subtle deficits at the less affected body side), there is converging evidence for more high-level deficits in prospective control, or motor planning, in particular, in individuals with right hemiparesis (Mutsaarts et al. 2005, 2006; Steenbergen et al. 2000a, 2004; te Velde et al. 2005).

These studies on manual control have advanced our knowledge about the different levels at which the motor system affects $\mathrm{CP}$ as well as the behavioural reorganisation during the performance of actions (e.g., van Roon et al. 
2005b). Yet, up to date, eye-hand coordination has not been investigated, despite preliminary evidence suggesting that it may contribute to deficits in action performance in individuals with CP (van der Meer et al. 1995; van der Weel et al. 1996). Even indirect evidence regarding the role of visual feedback for manual action performance in CP is scarce. Wann (1991) investigated visuo-proprioceptive integration in adolescents with CP. Comparing arm postures in a bimanual position-matching task under different conditions of sensory feedback, he found that, in contrast to adult controls, participants with $\mathrm{CP}$ employed very similar end postures (joint angle configurations) with the left and right arm in conditions in which vision of one or both arms was prevented. This was interpreted as evidence that they relied more strongly on purely proprioceptive information rather than on a shared visuospatial-proprioceptive representation and that the encoding of visual and proprioceptive information into a common egocentric frame may be problematic in these individuals. This would suggest that individuals with $\mathrm{CP}$ more strongly depend on continuous visual feedback for guiding manual actions to visual targets, compared to neurologically healthy adults.

Moreover, as the severity of the motor impairment, and concomitant proprioceptive impairment, increases from proximal to distal (e.g., Steenbergen et al. 2000b), it may be expected that actions with the affected-hand involving more fine motor control, such as grasping, need closer visual monitoring to provide additional feedback. In line with this, Steenbergen and colleagues (1996), studying bimanual actions in participants with hemiparetic $\mathrm{CP}$, made the anecdotal observation that (overt) visual attention seemed to be drawn to the affected side of the body. However, in contrast to the reasoning above, a recent study that scrutinized the effect of removing visual information of the moving limb on end-point accuracy in a straight-line drawing task (van Roon et al. 2005a) found no specific adverse effect of this manipulation in participants with tetraparetic cerebral palsy when using their relatively less-impaired hand compared to control participants. Thus, up to date, there is no consensus on the role of visual guidance for the control of manual actions in $\mathrm{CP}$.

Besides the sensorimotor impairments of the $\mathrm{AH}$, more high-level aspects of action control, such as motor planning and prospective control, have been shown to be compromised as well in hemiparetic CP. In research on motor planning in individuals with $\mathrm{CP}$, the LAH is used, as this hand has no (or small) movement restrictions, as is often the case with the AH. Hence, the higher-order process of motor planning can be studied without the confounding effects of possible movement restrictions. Atypical performance with the LAH was found for instance in tasks involving planning of the hand's posture at the end of the action (Steenbergen and Gordon 2006; Steenbergen et al. 2000a), in particular, in individuals with right hemiparesis (Steenbergen et al.
2004). In these tasks, individuals have to pick up objects and place them in another orientation on a designated target. Ample evidence in controls suggest that individuals pick-up objects with a grip that allows them to end the task with a comfortable posture, even when this means that they have to sacrifice comfort of the start grip (see Steenbergen and Gordon 2006). This "end state comfort" effect was not present in individuals with $\mathrm{CP}$, indicating a higher-level impairment of movement planning. Prospective control in individuals with hemiparetic CP was recently investigated in a collision avoidance task (te Velde et al. 2005). This study found later, hand-movement initiation in individuals with right as compared to left hemiparesis, again indicating reduced anticipation of action requirements. The extent to which deficits in motor planning or prospective control may be related to deviations in visual monitoring, with its typically anticipatory role for action control (Johansson et al. 2001) has not been investigated yet in individuals with CP.

In the present study, we examined eye-hand coordination in an unconstrained object prehension task in individuals with hemiparetic CP and neurologically healthy controls. To manipulate task difficulty, an obstacle was present in half of the trials, inducing a more complex transport trajectory. Participants performed the task with each hand separately. Based on the registration of eye and hand movements, we calculated several measures of temporal and spatial aspects of eye-hand coordination. Besides giving a first descriptive account of gaze patterns during object manipulation in a population with congenital hemiparesis, we addressed two hypotheses. Our first and main research question concerned the way in which individuals with hemiparetic CP adapt eye-hand coordination to the sensorimotor impairments of their affected hand. We hypothesized that individuals with $\mathrm{CP}$ would use increased visual monitoring when they perform the task with their $\mathrm{AH}$ as compared to performance with the LAH and to performance of control participants. Second, we examined eye-hand coordination when the task was performed with the LAH in participants with CP. Based on the previously established deficits in action planning, we hypothesized that gaze patterns are less anticipatory in general, in individuals with $\mathrm{CP}$. Given the limited knowledge on visumotor control in $\mathrm{CP}$ and on the role of eye-movements for action planning, this second research question was much more exploratory than the first.

\section{Methods}

\section{Participants}

In total, 16 individuals took part in the study. Six participants with hemiparetic $\mathrm{CP}$ constituted the experimental 
group and ten participants with no known history of neurological disorders served as controls. Control participants were all right handed (Dutch version of the Edinburgh handedness inventory, Oldfield 1971) University students (9 females, 20-25 years). The six participants of the experimental group (5 females, 14-19 years) were students at the Werkenrode Institute (Groesbeek, The Netherlands), where they followed an adapted educational programme. They had been asked to take part in the study based on their condition (viz. congenital spastic hemiparesis). Additional requirements were no known oculomotor disturbance (such as nystagmus), and having the motor, cognitive and attentional capacities to perform the experimental task as assessed by initial screening. All were able to walk and sit independently, and able to read and write with their LAH.

Consent was obtained prior to the experiment, both in verbal form from the participants during the initial screening session, as well as in written form from their parents or caregivers. In a separate session taking place after the experiment, the participants with $\mathrm{CP}$ performed two standardised dexterity tests, the Box and Block test for gross dexterity (Mathiowetz et al. 1985) and the Purdue pegboard test for fine dexterity (Tiffin 1968). One of the participants was not available for the manual dexterity testing afterwards. Table 1 shows the results of these tests as well as other participant information. For the Purdue-Pegboard test, the norm score for individuals at the age of 1617 years is 49.5 for the preferred hand. Standardised norm scores for the Box and Block test are not available for individuals younger than 20 years. For the youngest age group available, individuals between 20 and 24, the norm score is 88.2 for the preferred hand. The performance of the participants with $\mathrm{CP}$ was consistently below these norms, even when using the LAH.

All participants received the same reimbursement (€6 per hour) for taking part. The study was approved by the local ethics committee and performed in accordance with the standards laid down in the 1964 Declaration of Helsinki.

Table 1 Participant information for the participants with cerebral palsy

\begin{tabular}{lllll}
\hline Participant & Age & Paretic side & B and B ${ }^{\mathrm{a}, \mathrm{c}}$ & $\mathrm{PP}^{\mathrm{b}, \mathrm{c}}$ \\
\hline CP1 & 15 & Right & $15 / 38$ & $0 / 33$ \\
CP2 & 17 & Left & $24 / 52$ & $0 / 42$ \\
CP3 & 17 & Left & $16 / 32$ & $6 / 27$ \\
CP4 & 15 & Right & $15 / 43$ & $0 / 33$ \\
CP5 & 14 & Left & $18 / 42$ & $3 / 33$ \\
CP6 & 19 & Right & NA $^{\mathrm{d}}$ & NA $^{\mathrm{d}}$ \\
\hline
\end{tabular}

\footnotetext{
a Box and Blocks test

${ }^{\mathrm{b}}$ Purdue-Pegboard test

c Affected hand/less affected hand

${ }^{\mathrm{d}}$ Not available for testing
}

Setup and procedure

The experimental setup is depicted in Fig. 1. Participants were comfortably seated on a chair approximately centered at the midline of the table, which constituted the working region for the experiment. Two objects (blue and green), two target regions, and an optional obstacle were placed on the table, as well as a fixation target (see Fig. 1 and caption for details). Two loudspeakers, placed below the table, were used to verbally instruct the participant through a prerecorded voice. The experimenter sat facing the participant at a separate table. From this position he was able to supervise the experiment by instructing the participant, starting and terminating the trials, and monitoring participants' performance (directly and via a computer monitor that showed the eye movement recording system's output in real time).

For each participant, the experiment consisted of eight conditions in a $2 \times 2 \times 2$ factorial design, with the factors hand (AH/LAH for the participants with $\mathrm{CP}, \mathrm{NPH} / \mathrm{PH}$ for controls), obstacle (present, absent) and side (blue, green). In each of these conditions, five trials were performed, yielding a total of 40 trials per subject. The factors hand and obstacle were blocked while side was randomized within blocks. The order of the blocks was partially counterbalanced between participants. Prior to each trial, the participant was instructed to rest the "inactive" hand in his/ her lap, such that it did not interfere with the task.

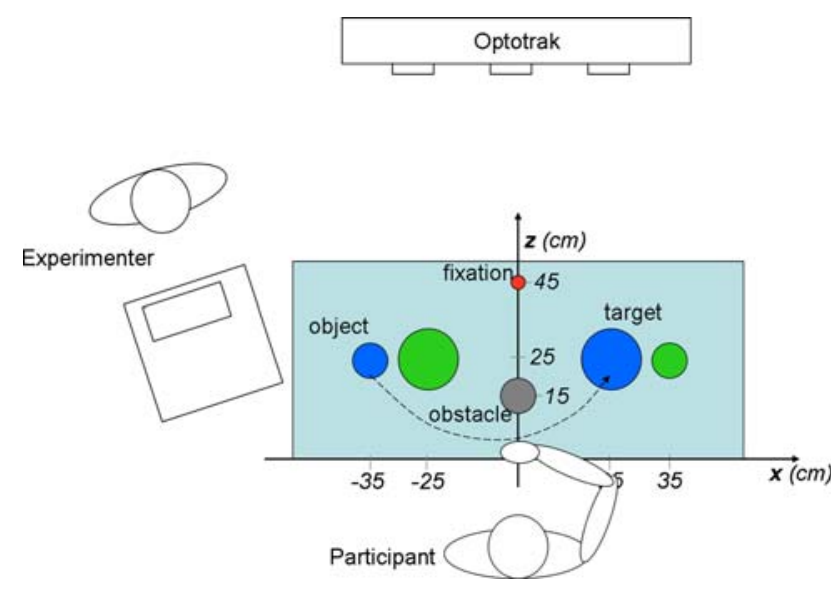

Fig. 1 Experimental setup (top view). The participant is seated at the table, with the right hand resting at the starting position. Positions of objects (blue and green cylinder, $6 \mathrm{~cm}$ in height, $3 \mathrm{~cm}$ in diameter, with an enlarged stabilizing basis of $6 \mathrm{~cm}$ in diameter), target regions (blue and green disc, $10 \mathrm{~cm}$ in diameter, contralateral to the corresponding object), optional obstacle (cylinder, $15 \mathrm{~cm}$ in height, $6 \mathrm{~cm}$ in diameter), and the pre-trial fixation target (red ball $2 \mathrm{~cm}$ in diameter mounted on a frame at about eye height) are shown. The experimenter was seated at a separate desk facing the participant. The dashed line represents the object transport movement from object to target region in a "blue" trial. The $x$ - and $z$-axis of the Optotrak system were approximately aligned to the axes indicated here, the $y$-axis was orthogonal to both (vertical axis) 
Each trial started with the task-hand resting at the starting position, and with gaze directed to the fixation target. After a variable delay (500-1,500 ms), the pre-recorded instruction "green" or "blue" (in Dutch) was played, upon which, the participant was to grasp the corresponding object and transport it to and place it on the contralateral target region of the same color. When an obstacle was present, the object had to pass in front of it, that is, between obstacle and participant (see Fig. 1, dashed arrow-line). After placing the object on the target region, the participant moved his/her hand back to the starting position and the experimenter put the cylinder back to its original location. When a trial failed, for instance because the cylinder slipped out of the participant's hand, it was immediately repeated. This never occurred more than twice in a single participant.

\section{Data acquisition}

During the experiment, hand and head movements were recorded using an Optotrak 3020 system (sampling rate set at $125 \mathrm{~Hz}$ ). Four Optotrak markers were located on the Eyelink helmet (see below) to monitor position and orientation of the head. Hand movements were recorded by means of a marker placed on the back, each of the participant's hands near the head of the second metacarpal. For two participants with $\mathrm{CP}$, this position did not guarantee sufficient visibility of the marker on the $\mathrm{AH}$ due to individual grasping patterns (excessive arm pronation leading to a thumbdown grasping posture). In these instances, the marker was placed more laterally on the hand. As we were mainly interested in the temporal aspects of the movements kinematics, this adjustment did not affect the measurement in a relevant way. The coordinate frame of the Optotrak system was oriented such that $x, y$ and $z$ axes roughly corresponded to the horizontal, vertical and posterior-anterior dimension with respect to the participant's position (see Fig. 1).

Gaze direction was assessed using a head mounted Eyelink II system (sampling frequency $500 \mathrm{~Hz}$ ), which was calibrated twice during the experiment, before each of the main blocks that corresponded to the obstacle present/absent conditions. To calibrate the system, the subject was asked to look at $10-15$ points covering the frontal working plane. These points were manually indicated by the experimenter by using a stick on the end of which both a fixation target (small red ball with a diameter of $13 \mathrm{~mm}$ ) and an Optotrak marker were attached. This "interactive" calibration method was chosen because pilot recordings revealed that two participants with CP failed to follow the instructions of the standard calibration procedure (employing a calibration board with LEDs). In addition, the "interactive" method allowed for constant monitoring of the Eyelink system's output by the experimenter (see "Setup and procedure").
The position of the fixation target and the position of the eye relative to the Eyelink helmet were used to compute gaze direction. Gaze direction was subsequently transformed to a $2 \mathrm{D}$ signal by projecting it to the $x y$-plane. Linear regression between this direction and the raw Eyelink data was used to calibrate the system. Although calibration error (deviation between actual and reconstructed gaze direction for the calibration targets) was relatively largeon average, $5^{\circ}$ of visual angle, increasing towards the periphery-it was well within the precision requirements needed to answer our research questions. Particular care was taken in the analysis of eye movements (see "Eye movements") to minimize the effect of this inaccuracy.

\section{Preprocessing}

The raw Optotrak data were partially interpolated with cubic spline interpolation (up to ten successive samples, corresponding to $80 \mathrm{~ms}$ ) and low-pass filtered (third order Butterworth filter with cut-off frequency of $10 \mathrm{~Hz}$ ). Hand velocity was computed by 3 -point numerical differentiation.

The raw Eyelink signal was transformed to relative gaze direction and integrated with the helmet position and orientation to yield absolute gaze direction. Approximate fixations were computed as the intersection of gaze direction with the plane parallel to the xy-plane (see Fig. 1) and containing the centers of objects and target regions. Only data from one eye (selected by visual inspection for each individual participant) were used for further analysis. Gaze data were partially interpolated (up to four successive samples, corresponding to $8 \mathrm{~ms}$, using cubic spline interpolation) and low-pass filtered (third order Butterworth with cut-off frequency of $20 \mathrm{~Hz}$ ). Subsequently, gaze velocity, acceleration, and jerk (second time differential of velocity) were computed by repeated 3-point numeric differentiation.

\section{Data reduction}

\section{Hand movements}

Hand movements were identified using an absolute velocity threshold $(0.20 \mathrm{~m} / \mathrm{s})$ and a direction criterion (start of a new movement indicated by a reversal of horizontal direction). These criteria provided the algorithms for the semi-automatic custom-written selection routines for the segmentation of the trials. Based on this segmentation, the grasp time (interval between the hand reaching and leaving the object region) and hand movement duration (interval between the hand leaving the object and reaching the target region) were determined. Analysis of eye-hand coordination was confined to the object transport phase that is from onset of the hand movement away from the object region till the end, reaching the target region. The duration of this movement was determined. 


\section{Eye movements}

Saccades were detected using a jerk (second time differential of velocity) criterion, which has been shown to be more reliable than methods based purely on gaze velocity or acceleration, especially for head-free eye movement recordings. A saccade was scored whenever jerk exceeded $200.000 \% \mathrm{~s}^{3}$, with an additional velocity requirement $(50 \%)$ (see Wyatt 1998, for similar analaysis). Saccade termination was determined as the first moment after saccade onset at which velocity dropped below $50 \%$. The total number of saccades registered in the experiment was 4,613. Saccade initiation/termination time was determined when none of the first or last eight samples $(16 \mathrm{~ms})$ were missing. Saccade peak velocity was determined, when in addition no more than four samples were missing in a row throughout the entire saccade. According to these criteria, saccade initiation and termination time could not be determined in $5.5 \%$ of all saccades, and peak velocity could not be determined in $10.5 \%$ of all saccades.

To test for potential group differences in basic eye movement kinematics, saccade main sequences were plotted, based on the saccades for which the peak velocity could be determined. Main sequences showed, for both groups, the typical linear relationship between amplitude and duration (e.g., Carpenter 1988), and the log-linear relationship between amplitude and peak velocity for all participants of both groups. No significant group differences were found in gradient or intercept of linear regressions of these relationships (all $p>0.05$, Welch's unpaired $t$-test).

Subsequently, for each trial the "object-leaving" and the "target-reaching" saccade were determined (in many trials these saccades coincided since there was a single gaze shift from object to target). Automatic detection of these saccades was complicated by the fact that precision of gaze data was not sufficient for a procedure based on landmark regions around object and target. Therefore, the following semi-automatic two-step procedure was adopted. First, fixations to object and target were determined automatically, assuming that these occurred before (up to $-600 \mathrm{~ms}$ ) the beginning and at the end of object transport. Fixation periods were defined as intervals of at least $200 \mathrm{~ms}$, during which, the standard deviation of absolute gaze fixation was smaller than $5 \mathrm{~mm}$. Note that this automatic procedure did not use absolute landmark regions but relative position information-this was possible because the object and target were placed at the lateral extremes of the working region of the experiment. For the obstacle, an analogous procedure was not possible.

Choices of this first step were inspected trial by trial and manually corrected if necessary. Obstacle fixations were not taken into account since it was not always possible to reliably distinguish them from other, frequently occurring intermediate fixations between object and target. Second, an automatic routine was used to detect the object-leaving saccade, defined as the first saccade, at the end of which, gaze had moved more than $10^{\circ}$ horizontally relative to the object fixation. Based on an analogous $10^{\circ}$ criterion, the target-reaching saccade was determined at the end of object transport. Thus, small $\left(<10^{\circ}\right)$ corrective saccades at the end of object transport were ignored in the definition of the target-reaching saccade.

\section{Eye-hand coordination}

Visual monitoring during object transport was quantified by the number of intermediate fixations, that is, the number of gaze shifts minus one occurring between the object-leaving and the target-reaching saccade. Note that this number does not include potential small corrective saccades at the end of object transport, since the target-reaching saccade was defined as the last saccade of horizontal amplitude $>10^{\circ}$ reaching the target region.

To examine temporal coordination between eye and hand at the beginning and end of object transport, two variables were computed: the movement onset asynchrony (MOA) and the movement termination asynchrony (MTA). The MOA was defined as the interval between the start of the object transport movement and the onset of the objectleaving saccade, with larger (positive) values corresponding to later gaze departure. Similarly, the MTA was determined by the delay between the termination of the object transport movement and the end of the target-reaching saccade, with smaller (negative) values corresponding to earlier target fixation. To eliminate a potential confounds with hand kinematics (in particular in the comparison of $\mathrm{AH}$ and LAH in participants with $\mathrm{CP}$ ), both MOA and MTA were normalized with respect to hand movement duration (expressed as percentage of the latter).

With respect to our experimental hypotheses, we predicted that closer visual monitoring would be reflected in greater gaze-hand proximity (smaller average angular distance), larger number of intermediate gaze fixations, and delayed gaze departure from the object region (that is, a longer $M O A$ ). Regarding prospective control, we assumed that less anticipatory gaze patterns would be reflected in delayed departure from the object region (longer MOA) and delayed arrival on target (shorter/less negative $M T A)$.

\section{Statistical analysis}

Data from one control participant were excluded due to insufficient quality of the Eyelink data, leaving data from six experimental and nine control subjects for statistical analysis. For each of the five variables of hand kinematics 
and eye-hand coordination introduced in "Data reduction", the means averaged across all replications in each condition were submitted to a three-way repeated measures ANOVA with one between-subject factor (group: $\mathrm{CP} /$ control) and two within-subject factors (hand: AH/LAH, NPH/PH; obstacle: present/absent). Subsequent analyses of simple effects were performed using paired $t$-tests for within-subject factors, and Welch's unpaired $t$-test (no equal variance assumed) for the between-subject factor. To correct for multiple (five) ANOVAs, the critical significance level was set to 0.01 .

\section{Results}

Of the total $15 \times 40=600$ trials, seven trials were entirely rejected due to insufficient data quality (no more than two trials in any individual participant).
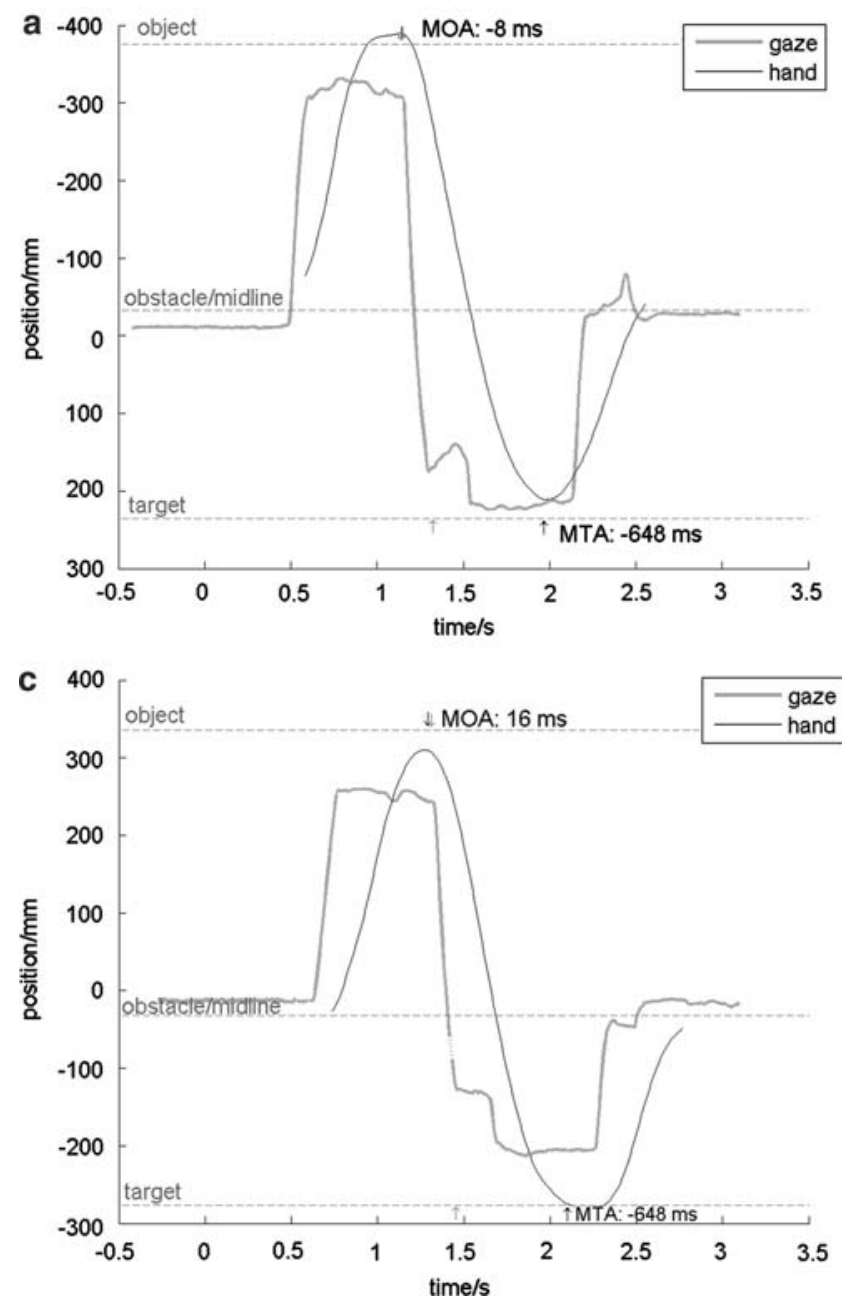

Fig. 2 Sample trials of two control participants, using their PH (a, c) and NPH $(\mathbf{b}, \mathbf{c})$ in trials with the obstacle present. Interpolated data points are represented by dashed lines. Vertical arrows at the top and
Sample trials

Figures 2 and 3 show representative trials from participants with CP and control participants. Horizontal components of head and hand position and gaze fixation (see "Data reduction") are plotted on the vertical axis, as a function of time. The intervals and durations of the (non-normalized) $M O A$ and MTA are indicated by vertical arrows and numerically in the plots. To allow comparisons between the plots, all data shown are from obstacle present trials. Visual inspection indicates qualitatively similar patterns (saccadic gaze shifts to future hand targets) in all conditions, and for both participant groups. Complete visual tracking of the trajectory of the hand was found only in a few trials (detected by visual inspection) in two participants with $\mathrm{CP}$ when using their $\mathrm{AH}$. For these trials, MOA and/or MTA could not be determined.

The representative trials shown in Fig. 2 (control participants using their PH and NPH) and Fig. 3 (participants with
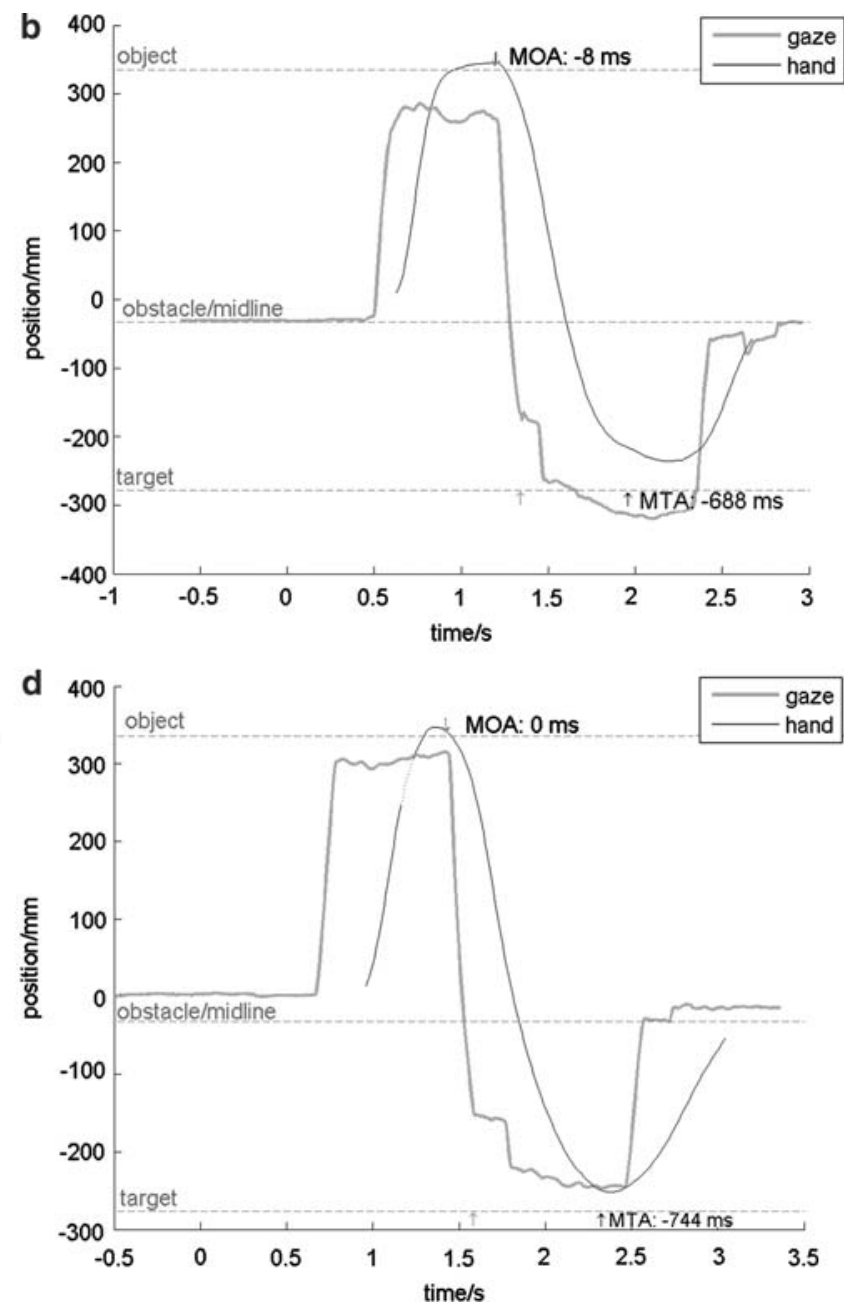

bottom of the plots specify the intervals defining the MOA and MTA (beginning/end of hand and eye movement), with numerical values of these measures added 

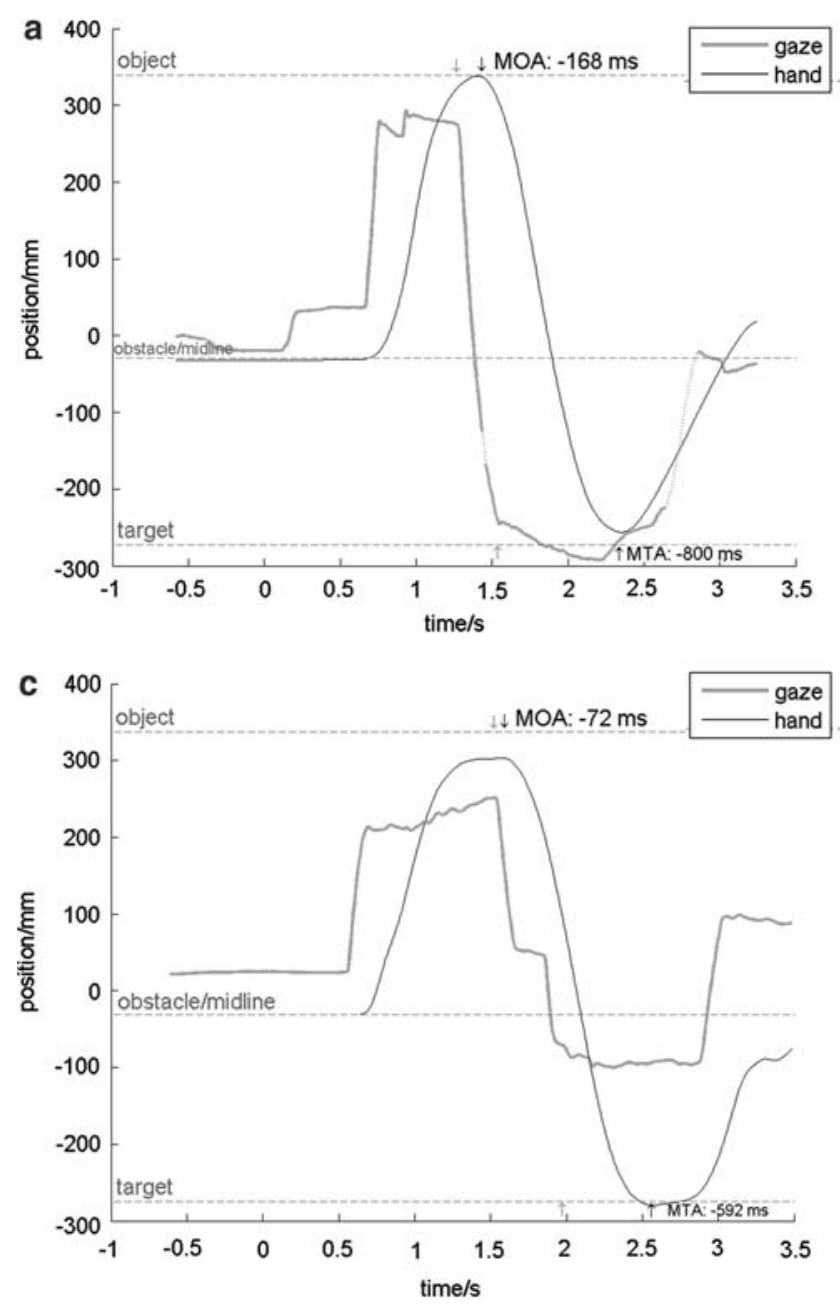

Fig. 3 Sample trials of two participants with CP, using their LAH (a, c) and $\mathrm{AH}(\mathbf{b}, \mathbf{d})$ in trials with the obstacle present. Note the delayed gaze departure (longer MOA) and increased number of intermediate fixations for the $\mathrm{AH}$ compared to the $\mathrm{LAH}$ and to both hands of control

$\mathrm{CP}$ using their $\mathrm{LAH}$ and $\mathrm{AH}$ ) illustrate some general trends when participants with $\mathrm{CP}$ use their $\mathrm{AH}$. These are a prolonged $M O A$, a larger number of intermediate fixations and a greater gaze-hand proximity compared to the other combinations of group and task hand. These observations are in agreement with the hypothesis of increased visual monitoring (see Eye-hand coordination"), and this is corroborated by the statistical analysis presented below. In contrast, no clear pattern with respect to the MTA is obvious from these data plots, nor from the statistical analysis.

\section{Dependent variables}

Values for the different dependent variables are summarized in Table 2. Due to missing samples, some of the measures reported below could not be determined in a number of trials. This number did not exceed 40 out of 600 trials
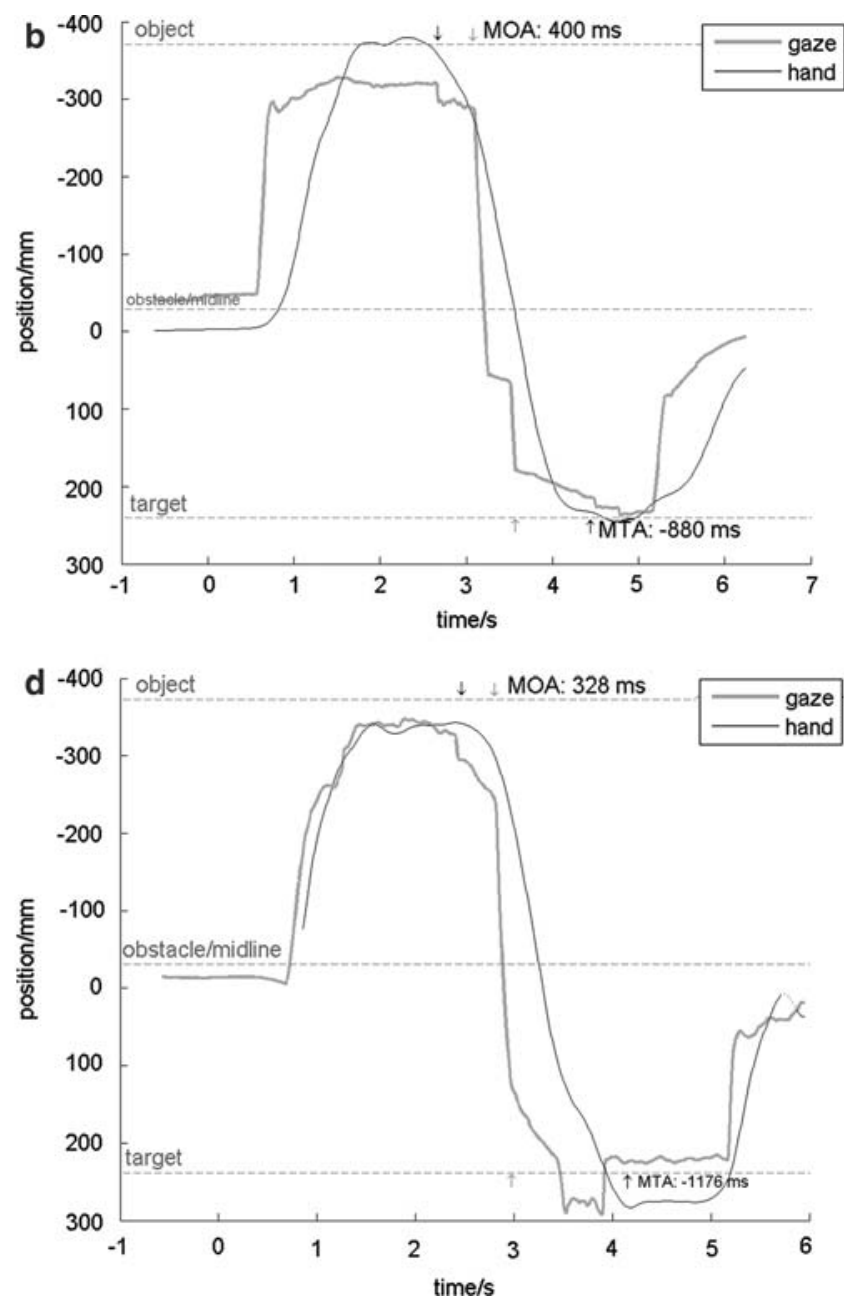

participants (Fig. 2). Interpolated data points are represented by dashed lines. Vertical arrows at the top and bottom of the plots specify the intervals defining the MOA and MTA (beginning/end of hand and eye movement), with numerical values of these measures added

(never more than five out of ten in a single condition and participant) for any of the variables.

Grasp time showed main effects of group, hand as well as a group-hand interaction (ANOVA, all $p$-values $<0.0001)$. Both, with the AH and the LAH participants with $\mathrm{CP}$ showed a longer grasp time than controls using either hand. The simple effect of hand was only present in participants with $\mathrm{CP}$ (longer grasp time with the AH compared to the LAH).

Hand movement durations for object transport showed main effects of group, hand, and obstacle, as well as a group-hand interaction (ANOVA, all $p$-values $<0.0001$ ). Simple effect analysis revealed that the effect of hand was present in both groups, but more pronounced in participants with CP. Moreover, the movement duration was longer in participants with $\mathrm{CP}$ and in the presence of an obstacle (Fig. 4). 
Table 2 Mean and standard deviation (between participants) of dependent variables

\begin{tabular}{|c|c|c|c|c|c|c|c|c|}
\hline \multirow[t]{3}{*}{ Dependent variable } & \multicolumn{4}{|c|}{ Participants with CP } & \multicolumn{4}{|c|}{ Control participants } \\
\hline & \multicolumn{2}{|l|}{$\overline{\mathrm{LAH}^{\mathrm{a}}}$} & \multicolumn{2}{|l|}{$\mathrm{AH}^{\mathrm{b}}$} & \multicolumn{2}{|l|}{$\overline{\mathrm{PH}^{\mathrm{c}}}$} & \multicolumn{2}{|l|}{$\mathrm{NPH}^{\mathrm{d}}$} \\
\hline & No obstacle & Obstacle & No obstacle & Obstacle & No obstacle & Obstacle & No obstacle & Obstacle \\
\hline \multicolumn{9}{|c|}{ Hand movement duration (s) } \\
\hline Mean & 0.81 & 1.05 & 1.05 & 1.60 & 0.61 & 0.83 & 0.65 & 0.87 \\
\hline SEM & 0.03 & 0.05 & 0.03 & 0.15 & 0.01 & 0.02 & 0.02 & 0.03 \\
\hline \multicolumn{9}{|l|}{ Object grasp time } \\
\hline Mean & 0.22 & 0.25 & 1.07 & 1.16 & 0.063 & 0.071 & 0.086 & 0.094 \\
\hline SEM & 0.03 & 0.04 & 0.14 & 0.16 & 0.009 & 0.01 & 0.017 & 0.015 \\
\hline \multicolumn{9}{|c|}{ Number of intermediate fixations } \\
\hline Mean & 0.24 & 0.82 & 0.76 & 1.30 & 0.06 & 0.73 & 0.07 & 0.61 \\
\hline SEM & 0.09 & 0.34 & 0.24 & 1.21 & 0.04 & 0.22 & 0.06 & 0.26 \\
\hline \multicolumn{9}{|c|}{ Movement onset asynchrony ( $\%$ hand movement duration) } \\
\hline Mean & -6.88 & 1.53 & 13.4 & 14.62 & -4.45 & 0.02 & -3.14 & 3.84 \\
\hline SEM & 5.81 & 4.96 & 4.37 & 2.12 & 4.84 & 4.21 & 4.84 & 4.37 \\
\hline \multicolumn{9}{|c|}{ Movement termination asynchrony ( $\%$ hand movement duration) } \\
\hline Mean & -76.8 & -61.2 & -49.5 & -51.4 & -75.7 & -65.6 & -71.5 & -62.2 \\
\hline SEM & 5.39 & 6.87 & 2.84 & 3.64 & 4.39 & 3.78 & 4.64 & 3.56 \\
\hline
\end{tabular}

${ }^{\text {a }}$ Less affected hand

${ }^{b}$ Affected hand

c Preferred hand

${ }^{\mathrm{d}}$ Non-preferred hand

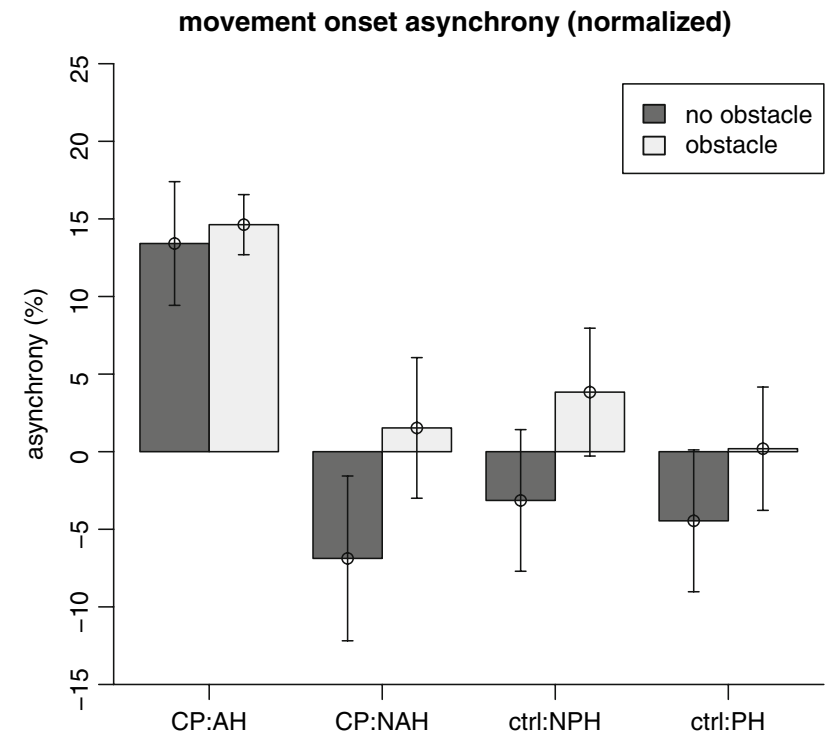

Fig. 4 Mean \pm SEM of the movement onset asynchrony (MOA) as a function of participant group, task hand and obstacle presence. MOA was normalized with respect to hand movement duration

Regarding the number of intermediate fixations during object transport, a main effect of obstacle $[F(1,13)=15.06$, $p<0.002]$ was found, as well as a group-hand interaction $[F(1,13)=12.36, p<0.005]$. Analysis of simple effects showed that the number of intermediate fixations was larger in the obstacle present condition than in the obstacle absent condition. With respect to the group-hand interaction, significant differences were found between participants with $\mathrm{CP}$ using their $\mathrm{AH}$ and control participants using either hand (higher number of intermediate fixations for $\mathrm{CP}$ using $\mathrm{AH}$ ), but not between the AH versus LAH of participants with CP.

Temporal eye-hand coordination at the beginning of object transport, as quantified by MOA (see Fig. 4), showed main effects of hand $[F(1,13)=17.25, p<0.002]$ and a group-hand interaction $[F(1,13)=12.55, p<0.005]$. The simple effect of hand was present in participants with $\mathrm{CP}$, with longer MOA in the AH condition, but not in control participants. Moreover, there was a significant difference (prolonged $M O A$ ) between participants with $\mathrm{CP}$ using their $\mathrm{AH}$ and control participants using either hand.

With respect to $M T A$, a main effect of hand $[F(1,13)=$ $25.68, p<0.0005]$, a group-hand interaction $[F(1,13)=$ $14.189, p<0.005$ ] were found, as well as a marginally significant group-hand-obstacle interaction $[F(1,13)=8.19$, $p=0.013]$. The simple effect of hand was present only in participants with $\mathrm{CP}$, with a shorter (less negative) MTA when using the $\mathrm{AH}$. Also the difference between participants with $\mathrm{CP}$ using the $\mathrm{AH}$ and control participants using either hand was significant (shorter MTA in participants with $\mathrm{CP}$ using the $\mathrm{AH}$ ). 


\section{Discussion}

The main question we pursued in the present study was whether and in which way individuals with hemiparetic CP adapt eye-hand coordination to their sensorimotor impairments, in particular, when actions are performed with the affected hand (AH). We hypothesized that participants with $\mathrm{CP}$ would more closely monitor actions performed with their $\mathrm{AH}$, compared to actions performed with their less affected hand (LAH) and compared to neurologically healthy control participants using either hand. Qualitatively, eye-hand coordination patterns were similar among control participants and participants with $\mathrm{CP}$, regardless of the hand used to perform the task. That is, anticipatory saccadic gaze shifts were used to fixate future "action sites"- - such as object, target, or intermediate fixations-in advance. Notwithstanding these qualitative resemblances, a more fine-grained analysis of temporal and spatial aspects of eye-hand coordination did confirm our hypothesis of increased visual monitoring when moving with the AH. Depending on the measure employed, this effect was present when comparing performance with the $\mathrm{AH}$ to control participants using either hand (increased number of intermediate fixations), or in addition for comparing performance with the $\mathrm{AH}$ to performance with the $\mathrm{LAH}$ in participants with $\mathrm{CP}$ (delayed gaze departure from object and hand, i.e., a longer MOA).

It is important to note that our main result regarding visual monitoring is an interaction effect of group and task hand (effect of task hand was present only in the experimental group). Therefore, it cannot be explained by potential group differences in oculomotor control or gaze data quality. Moreover, despite previous findings that did not find a strong relationship between gaze-exit time relative to hand action and task speed (Flanagan and Johansson 2003), we decided to normalize our measures of temporal eyehand coordination in order to exclude any potential confounding effects of hand kinematics. This normalization may be considered rather conservative. Without normalizing, the effects of task and participant group on MOA may have even been stronger.

A second, and more speculative hypothesis of the present study was that in general, gaze patterns might be less anticipatory in participants with $\mathrm{CP}$, contributing to deficits in prospective control and action planning that have been reported in this population (Steenbergen et al. 2000a, 2004; te Velde et al. 2005), in particular, individual with right hemiparesis. The present study found no evidence in support of this hypothesis. Although participants with CP did show a prolonged $M O A$, that is, a delayed gaze departure that may indicate less anticipation, this effect was restricted to the AH. No significant differences in the MOA were found between actions of participants with $\mathrm{CP}$ using their
LAH and control participants using their PH. Similarly, for our measure of temporal eye-hand coordination at the end of object transport (the movement termination asynchrony, MTA), a delayed gaze arrival on target was only present in participants with $\mathrm{CP}$ using their $\mathrm{AH}$, not as a general group effect. In addition, preliminary analyses had shown no difference between individuals with left and right hemiparesis with respect to any of our dependent variables. Thus our results suggest that deficits in prospective action control found in CP are not directly related to atypical eye-hand coordination. However, this statement is made with caution as we only tested a small number of participants $(n=6$ in total, $n=3$ for each left and right hemiparesis).

An unexpected finding of the present study was that gaze frequently departed from the object region only after the hand movement had started, in particular, in the presence of the obstacle. This was also the case in control participants and is in contrast to the results of Johansson et al. (2001), who reported that in the majority of the trials gaze departure from the grasp site occurred even before the fingertips had reached the object. These contrasting findings may in part be explained by differences in the experimental set-up. In the present study the distance between initial object position and the target was much larger compared to the one used by Johansson et al. (2001), leading to longer hand movement durations. This potential confounding effect of hand movement duration needs to be addressed in future research, for instance, by systematically manipulating hand movement distance in order to disentangle components of online and prospective control in the MOA. Also, task complexity might play a role. In a block-stacking task, Flanagan and Johansson (2003) found gaze to depart on average just before hand movement onset, which is more consistent with our present findings.

In sum, we found evidence in support the hypothesis of increased visual monitoring of manual actions of individuals with hemiparetic $\mathrm{CP}$ performing actions with their $\mathrm{AH}$, both at the beginning and during object transport. This may reflect a strategy of visual compensation for sensorimotor deficits (see also, Mulder et al. 2001). However, since the functional significance of the observed gaze patterns was not addressed in the present study, the question whether and by which neurophysiological mechanisms vision enhances manual performance requires further research. Generalizability of our results is certainly limited by the fact that our experimental group was relatively small $(n=6)$ and highly selective, considering the high variability in the $\mathrm{CP}$ population regarding intellectual and motor abilities. In particular, the null-findings on anticipatory control and the comparison of individuals with left and right hemiparesis should be considered preliminary and require further investigation. On the other hand, the fact that we did find significant effects with respect to online 
visual control (number of intermediate fixations and MOA) despite the small number of participants suggests that the observed effects are robust.

Acknowledgments We would like to thank Michel Bex for invaluable help with running the experiment. Stan van Pelt, Hein van Schie and Ruud Meulenbroek are acknowledged for their useful suggestions, and Wilfried Nillesen from the Werkenrode Institute for organizational support.

Open Access This article is distributed under the terms of the Creative Commons Attribution Noncommercial License which permits any noncommercial use, distribution, and reproduction in any medium, provided the original author(s) and source are credited.

\section{References}

Albright AL (1996) Spasticity and movement disorders in cerebral palsy. J Child Neurol 11:S1-S4

Bax M, Goldstein M, Rosenbaum P, Leviton A, Paneth N (2005) Proposed definition and classification of cerebral palsy, April 2005introduction. Dev Med Child Neurol 47(8):571-576

Bekkering H, Sailer U (2002) Commentary: coordination of eye and hand in time and space. Prog Brain Res 140:365-373

Carpenter R (1988) Movements of the eyes. Pion, London

Cooper J, Majnemer A, Rosenblatt B, Birnbaum R (1995) The determination of sensory deficits in children with hemiplegic cerebralpalsy. J Child Neurol 10(4):300-309

Eliasson AC, Gordon AM, Forssberg H (1991) Basic coordination of manipulative forces of children with cerebral palsy. Dev Med Child Neurol 33(8):661-670

Flanagan JR, Johansson RS (2003) Action plans used in action observation. Nature 424(6950):769-771

Freund HJ (1987) Abnormalities of motor behaviour after cortical lesions in human. In: Mountcastle VB (ed) Handbook of physiology, section 1 , the nervous system. William and Wilkins, Baltimore, pp 763-810

Gordon AM, Charles J, Steenbergen B (2006) Fingertip force planning during grasp is disrupted by impaired sensorimotor integration in children with hemiplegic cerebral palsy. Pediatr Res 60(5):587-591

Grossberg S, Kupperstein M (1989) Neural dynamics of adaptive sensory-motor control (Expanded edn) Pergamon, New York

Hayhoe M, Ballard D (2005) Eye movements in natural behavior. Trends Cogn Sci 9(4):188-194

Johansson RS, Westling GR, Backstrom A, Flanagan JR (2001) Eyehand coordination in object manipulation. $\mathrm{J}$ Neurosci 21(17):6917-6932

Kwong KL, Wong YC, Fong CM, Wong SN, So KT (2004) Magnetic resonance imaging in 122 children with spastic cerebral palsy. Pediatr Neurol 31(3):172-176

Mathiowetz V, Volland G, Kashman N, Weber K (1985) Adult norms for the box and block test of manual dexterity. Am J Occup Ther 39(6):386-391
Mulder T, den Otter R, van Engelen B (2001) The regulation of fine movements in patients with Charcot Marie Tooth, type Ia: some ideas about continuous adaptation. Motor Control 5(2):200-214

Mutsaarts M, Steenbergen B, Bekkering H (2005) Anticipatory planning of movement sequences in hemiparetic cerebral palsy. Motor Control 9(4):439-458

Mutsaarts M, Steenbergen B, Bekkering H (2006) Anticipatory planning deficits and task context effects in hemiparetic cerebral palsy. Exp Brain Res 172(2):151-162

Oldfield RC (1971) Assessment and analysis of handedness-Edinburgh inventory. Neuropsychologia 9(1):97

Sailer U, Flanagan JR, Johansson RS (2005) Eye-hand coordination during learning of a novel visuomotor task. J Neurosci 25(39):8833-8842

Steenbergen B, Gordon AM (2006) Determinants for activity limitations in cerebral palsy: converging evidence for disorders in motor planning. Dev Med Child Neurol 48:780-783

Steenbergen B, Meulenbroek R. (2006) Deviations in upper-limb function of the less-affected side in congenital hemiparesis. Neuropsychologia 44:2296-2307

Steenbergen B, Hulstijn W, deVries A, Berger M (1996) Bimanual movement coordination in spastic hemiparesis. Exp Brain Res 110(1):91-98

Steenbergen B, Hulstijn W, Dortmans S. (2000a) Constraints on grip selection in cerebral palsy-minimising discomfort. Exp Brain Res 134(3):385-397

Steenbergen B, van Thiel E, Hulstijn W, Meulenbroek RGJ (2000b) The coordination of reaching and grasping in spastic hemiparesis. Hum Mov Sci 19(1):75-105

Steenbergen B, Meulenbroek RGJ, Rosenbaum DA (2004) Constraints on grip selection in hemiparetic cerebral palsy: effects of lesional side, end-point accuracy, and context. Cogn Brain Res 19(2):145159

te Velde AFT, van der Kamp J, Becher JG, van Bennekom C, Savelsbergh GJP (2005) Planning and control in a manual collision avoidance task by children with hemiparesis. Motor Control 9(4):417-438

Tiffin J (1968) Purdue pegboard examiner's manual. Science Research Associates, Chicago

van der Meer ALH, van der Weel FR, Lee DN, Laing IA, Lin JP (1995) Development of prospective control of catching moving-objects in preterm at-risk infants. Dev Med Child Neurol 37(2):145-158

van der Weel FR, Van der Meer ALH, Lee DN (1996) Measuring dysfunction of basic movement control in cerebral palsy. Hum Mov Sci 15(2):253-283

van Roon D, Steenbergen B, Meulenbroek RGJ (2005a) Movementaccuracy control in tetraparetic cerebral palsy: Effects of removing visual information of the moving limb. Motor Control 9(4):372-394

van Roon D, Steenbergen B, Meulenbroek RGJ (2005b) Trunk use and co-contraction in cerebral palsy as regulatory mechanisms for accuracy control. Neuropsychologia 43(4):497-508

Wann JP (1991) The Integrity of visual-proprioceptive mapping in cerebral-palsy. Neuropsychologia 29(11):1095-1106

Wyatt HJ (1998) Detecting saccades with jerk. Vision Res 38(14):2147-2153 